\title{
SCOTROC 2A: Carboplatin followed by docetaxel or docetaxel- gemcitabine as first-line chemotherapy for ovarian cancer
}

\author{
PA Vasey*, I,9, R Atkinson², R Osborne ${ }^{3}$, D Parkin ${ }^{4}$, R Symonds $^{5}$, J Paul', L Lewsley', R Coleman ${ }^{6}$, NS Reed', \\ S Kaye ${ }^{7}$ and GJS Rustin ${ }^{8}$
}

'CR-UK Clinical Trials Unit, Beatson Oncology Centre, Western Infirmary, Dumbarton Road, Glasgow GII 6NT, Scotland, UK; ${ }^{2}$ Belfast City Hospital HSS Trust, 5 I Lisburn Road, Belfast BT9 7AB, UK; ${ }^{3}$ Dorset Cancer Centre, Poole Hospital NHS Trust, Longfleet Road, Poole, Dorset BHI 5 2JB, UK; ${ }^{4}$ Aberdeen Royal Infirmary, Foresterhill, Aberdeen AB25 2ZN, UK; ${ }^{5}$ University Hospitals of Leicester NHS Trust, Leicester Royal Infirmary, Infirmary Square, Leicester LEI 5WW, UK; ${ }^{6}$ Weston Park Hospital NHS Trust, Whitham Road, Sheffield SIO 2SJ, UK; ${ }^{7}$ Royal Marsden Hospital, Downs Road, Sutton, Surrey SM2 5PT, UK; ${ }^{8}$ Mount Vernon Hospital, Rickmansworth Road, Northwood, Hertfordshire HA6 2RN, UK

The feasibility of sequential carboplatin followed by docetaxel-based therapy for untreated ovarian cancer was determined. Patients received four q3w cycles of carboplatin AUC 7, then four q3w cycles of either docetaxel $100 \mathrm{mg} \mathrm{m}^{-2}$ (day I) (arm A); docetaxel $75 \mathrm{mg} \mathrm{m}^{-2}$ (day 8) and gemcitabine $1250 \mathrm{mg} \mathrm{m}^{-2}$ (days l,8) (arm B) or docetaxel $25 \mathrm{mg} \mathrm{m}^{-2}$ and gemcitabine $800 \mathrm{mg} \mathrm{m}^{-2}$ (both given weekly (days I,8, I 5)) (arm C). A total of 44 patients were randomised to each treatment arm. None of the arms demonstrated an eight cycle completion rate $(70.5 / 72.7 / 45.5 \%$ in arms $A / B / C$, respectively), which was statistically greater than $60 \%(P=0.102$, $P=0.056, P=0.982$ ) which was our formal feasibility criteria, although only the completion rate in arm $C$ was clearly worse than this level. The overall response rate (ORR) after carboplatin was $65.7 \%$ in 70 evaluable patients. In evaluable patients, ORRs after docetaxel-based cycles were: arm A 84.0\% (2I out of 25); arm B 77.3\% (I7 out of 22); arm C 69.6\% (I6 out of 23). At follow-up (median 30 months), median progression-free survival times were: arm A 15.5 months (95\% Cl: 10.5-20.6); arm B I8.I months (95\% Cl: 15.9-20.3); arm C, 13.7 months (95\% Cl: 12.8-14.6). Neutropenia was the predominant grade 3-4 haematological toxicity: $77.8 / 85.7 / 54.4 \%$ in arms $A / B / C$, respectively. Dyspnoea was markedly increased in both gemcitabine-containing arms $(P=0.00 \mathrm{I})$ but was worse in arm C. Although just failing to rule out eight cycle completion rates less than $60 \%$, within the statistical limitations of these small cohorts, the overall results for arms A and B are encouraging. Larger phase III studies are required to test these combinations.

British Journal of Cancer (2006) 94, 62-68. doi:I0.1038/sj.bjc.6602909 www.bjcancer.com (c) 2006 Cancer Research UK

Keywords: ovarian cancer; docetaxel; carboplatin; gemcitabine; triple-agent therapy; sequential therapy

Primary cytoreductive surgery plus chemotherapy is the standard treatment for patients with advanced epithelial ovarian cancer, with paclitaxel-carboplatin now considered the treatment of choice for first-line chemotherapy (Kaye, 2000). However, despite a modest improvement in survival in recent years, the median 5 -year survival for advanced-stage disease remains poor at $31 \%$ (American Cancer Society, 2004).

Peripheral neuropathy is a common adverse event associated with paclitaxel-based therapy (Guastalla and Dieras, 2003) and can lead to early treatment discontinuation. Docetaxel is a secondgeneration taxane that has pharmacological and pharmacokinetic

\footnotetext{
*Correspondence: Dr PA Vasey; E-mail: Paul_Vasey@health.qld.gov.au

${ }^{9}$ Present address: University of Queensland, Division of Medicine, Brisbane, Australia, Q4029

P Vasey, R Atkinson, R Osborne, D Parkin, S Paul, R Coleman, J Paul, EA Lewsley, S Kaye, R Gordon, for the Scottish Gynaecological Cancer Trials Group (2003). Carboplatin (Cb) followed sequentially by docetaxel (D) \pm gemcitabine $(\mathrm{G})$ in ovarian, peritoneal and fallopian tube cancers: results of SCOTROC 2A. Proc Am Soc Clin Oncol 22: 449 (abstract 1804).

Revised II November 2005; accepted I 8 November 2005
}

advantages over paclitaxel (Gligorov and Lotz, 2004) and a substantially different toxicity profile to paclitaxel when combined with carboplatin (Hsu et al, 2004). Like paclitaxel, docetaxel has significant activity in ovarian cancer (Mäenpää, 2003) and recently the first Scottish Randomised Trial in Ovarian Cancer (SCOTROC 1) established that docetaxel - carboplatin provides similar survival, less neurotoxicity and greater improvement in some quality of life (QoL) parameters compared with paclitaxel-carboplatin (Vasey et al, 2004).

The addition of a third cytotoxic agent to a platinum-taxane doublet may potentially improve outcomes in ovarian cancer. High response rates have been reported when gemcitabine is added to a paclitaxel-platinum combination for advanced ovarian cancer (Hansen, 2002), albeit at the expense of significant haematological toxicity (Berkenblit et al, 2003; Barlow et al, 2004). Likewise, addition of pegylated liposomal doxorubicin (PLD) to frontline carboplatin-paclitaxel as a concurrent triplet necessitates significant PLD dose reduction (Bookman, 2003). Strategies that may potentially reduce such toxicities from triple-agent regimens include sequential scheduling or the use of alternating or sequential doublets. All these are currently being evaluated as an alternative to paclitaxel-carboplatin in the recently completed 
five-arm trial conducted by the Gynaecologic Oncology Group (GOG) and the International Collaborative Ovarian Neoplasm (ICON) group (GOG-182/ICON5). Survival data are not expected to be reported before 2006 .

A sequential-type approach may not only reduce toxicity but might also help to overcome any antagonism which may exist between agents - one possible reason for the failure of paclitaxelcarboplatin to improve survival over carboplatin alone in the ICON3 trial (International Collaborative Ovarian Neoplasm Group, 2002). Further support for a sequential approach is based on studies which show that ovarian cancer cells with abrogated $p 53$ gene function are sensitised to taxanes (Cassinelli et al, 2001), and that ovarian tumours with mutated $p 53$ are more responsive to taxanes and less responsive to platinum agents than wild-type $p 53-$ expressing tumours (Lavarino et al, 2000; Reles et al, 2001). Thus, initial platinum treatment might eradicate one tumour population of wild-type $p 53$ cells, leaving a population of predominantly mutant p53 cells amenable to treatment with taxanes. In the clinical setting, results from trial GOG-132 suggest that platinum therapy followed - prior to clinical progression - by paclitaxel does not result in loss of efficacy when compared with concurrent administration (Muggia et al, 2000). This randomised study was designed to assess the feasibility of sequentially scheduling singleagent carboplatin followed by docetaxel alone or a docetaxelgemcitabine doublet as first-line therapy for ovarian cancer.

\section{PATIENTS AND METHODS}

\section{Patients}

Female patients aged $\geqslant 18$ years with histologically confirmed epithelial ovarian carcinoma, fallopian tube cancer or ovarian-type primary peritoneal cancer were eligible for the study. Additional inclusion criteria were: International Federation of Gynecologic Oncology (FIGO) stages Ic-IV; Eastern Cooperative Oncology Group performance status $\leqslant 2$; no prior chemotherapy or radiotherapy; adequate bone marrow, hepatic and renal function. Exclusion criteria included: mixed mesodermal tumours; tumours considered borderline or 'possibly malignant'; concurrent malignancy or malignancy within the previous 5 years (except curatively treated uterine cervical carcinoma in situ or basal cell skin carcinoma); history of prior serious allergic reactions; pregnancy or lactation; symptomatic peripheral neuropathy $\geqslant$ grade 2 .

The study had central International Review Board approval and all patients gave written informed consent. Randomisation took place within 8 weeks of initial surgery (laparotomy/biopsy) and patients were allocated to treatment using a minimising algorithm with the following factors: extent of residual disease; centre; FIGO stage; performance status; tumour grade; pretreatment CA-125; presence or absence of primary peritoneal or fallopian tube cancer; interval debulking intention.

\section{Treatment}

Eight cycles (24 weeks) of chemotherapy were planned at 3-weekly intervals. After randomisation, all eligible patients received four cycles of carboplatin AUC (area under the curve) 7 (1-h infusion) on day 1 of each cycle. The dose of carboplatin was derived via the Calvert formula $(\mathrm{mg}=($ glomerular filtration rate +25$) \times 7)$ (Calvert et al, 1989) and by using ${ }^{51} \mathrm{Cr}$ EDTA (edetic acid) measurement of glomerular filtration rate (Chantler et al, 1969). This dose remained fixed for all cycles unless toxicity mandated dose reduction.

Patients then received either four cycles of docetaxel $100 \mathrm{mg} \mathrm{m}^{-2}$ (1-h infusion) on day 1 ( $\operatorname{arm~A);~four~cycles~of~docetaxel~}$ $75 \mathrm{mg} \mathrm{m}^{-2}$ (1-h infusion) on day 8 plus gemcitabine $1250 \mathrm{mg} \mathrm{m}^{-2}$ (30-min infusion) on days 1 and 8 (arm B); four cycles of docetaxel
$25 \mathrm{mg} \mathrm{m}^{-2}$ plus gemcitabine $800 \mathrm{mg} \mathrm{m}^{-2}$, both given as $30-\mathrm{min}$ infusions on days 1,8 and 15 ( $\operatorname{arm~C}$ ). Gemcitabine was always administered after docetaxel.

Premedication for docetaxel comprised dexamethasone $8 \mathrm{mg}$ twice daily for 3 days starting the day before docetaxel administration; oral antiemetics included granisetron $1 \mathrm{mg}$ prechemotherapy and domperidone $20 \mathrm{mg}$ up to four times daily if required.

Cycles were repeated in the absence of progressive disease or prohibitive toxicity. Interval debulking surgery was permitted after finishing carboplatin treatment and before starting docetaxelbased therapy (provided chemotherapy was restarted within 3 weeks).

\section{Dose/schedule modifications}

Treatment was delayed for up to 2 weeks if the neutrophil count was $<1.5 \times 10^{9} 1^{-1}$ or the platelet count was $<100 \times 10^{9} 1^{-1}$. Prophylactic antibiotics were recommended for subsequent cycles following grade 4 febrile neutropenia. This was treated by standard protocols involving hospital admission, intravenous antibiotics and (if required) the use of granulocyte-colony-stimulating factors.

The following dose reductions were indicated at first occurrence of neutrophils $<1.5 \times 10^{9} 1^{-1}$ or platelets $<100 \times 10^{9} 1^{-1}$ lasting $>1$ week but $<2$ weeks, neutropenic fever, or complicated grade 4 thrombocytopenia: carboplatin reduction to AUC 6 (all arms), and a docetaxel reduction to $75 \mathrm{mg} \mathrm{m}^{-2}$ in arm A or a $25 \%$ reduction for both gemcitabine and docetaxel in arms B and C. At the second occurrence of these haematological toxicities, carboplatin was reduced to AUC 5, docetaxel reduced to $60 \mathrm{mg} \mathrm{m}^{-2}$ (arm A), and both drugs reduced by a further $25 \%$ (arms B/C). For grade 3-4 nonhaematological toxicity during docetaxel-based cycles, treatment was delayed until toxicity reversed to $\leqslant$ grade 1 ; the doses on subsequent cycles were then reduced by $25 \%$. Treatment delay for $\leqslant 2$ weeks was allowed for mucositis grade $\geqslant 3$, painful or troublesome mucositis grade 2 and skin toxicity grade $\geqslant 2$; subsequent doses were reduced by $25 \%$. In an attempt to improve on the initially poor dose intensity observed in arm C, the protocol was amended in June 2001 to lower the threshold for dose reductions to a neutrophil count of $<1.0 \times 10^{9} 1^{-1}$ or a platelet count of $<75 \times 10^{9} 1^{-1}$, with delays for complicated thrombocytopenia mandated until platelets were $\geqslant 75 \times 10^{9} 1^{-1}$.

For significant hypersensitivity reactions to docetaxel, the infusion was stopped, symptoms were treated and patients rechallenged within $3 \mathrm{~h}$ without further premedication (if appropriate). Milder reactions were managed by slowing the infusion rate, observation until recovery and then reinfusion at the initial rate.

\section{Clinical assessments}

Before study entry, patients underwent a physical examination, abdomino-pelvic computed tomography scan, electrocardiogram, chest X-ray, CA-125, full biochemical profile, full blood count and glomerular filtration rate measurement. Blood counts and biochemical profiles were taken at the start of each carboplatin cycle and weekly during docetaxel-based treatment. CA-125 level was measured at the start of each cycle. Efficacy was assessed using modified Southwest Oncology Group Solid Tumour Response Criteria and by CA-125 (Rustin et al, 1996). Quality of life was evaluated before starting treatment and before each treatment cycle using the European Organisation for Research and Treatment of Cancer questionnaires QLQ-C30 and QLQ-OV28 (Cull et al, 2001). Toxicities were documented throughout chemotherapy using the National Cancer Institute Expanded Common Toxicity Criteria (NCI-CTC, Version 2.0). Neurotoxicity was assessed using a structured neurological questionnaire and examination before study entry and after four and eight cycles (Cassidy et al, 1998). 
All patients were followed up every 2 months until progressive disease was documented. Neurological and QoL assessments were continued every 4 months for up to 2 years.

\section{Statistical considerations}

The primary end point for this randomised feasibility study was the percentage of patients completing eight cycles of chemotherapy within each treatment arm. A completion rate of $\geqslant 80 \%$ was deemed clearly acceptable, $60-80 \%$ was deemed a 'grey area' and $\leqslant 60 \%$ was deemed clearly unacceptable. The study was designed to test the null hypothesis that the completion rate was $\leqslant 60 \%$ against the alternative that it was $>60 \%$. The one-sided significance level was set at $5 \%$ and the power of the study when the true completion rate was $80 \%$ was set at $90 \%$; this required 44 patients to be recruited to each treatment arm. The study was not powered to detect significant differences in efficacy between the three arms; randomisation was utilised in order to ensure that patients with similar characteristics received each of the three options. However, it also permitted a preliminary analysis to be made of the range of efficacy to be expected.

Protocol-defined secondary end points were: toxicities, QoL, clinical response rates and CA-125 responses, and progression-free and overall survival (in particular, at 8 months) (end of treatment). Toxicities and changes in QoL during taxane-based therapy were compared between arms using the Mann-Whitney $U$-test. Quality of life end points were examined in two sets: global health status and functional scales, and symptom scales (including fatigue, nausea and vomiting, pain, abdominal/gastrointestinal symptoms, peripheral neuropathy and hair loss). The statistical significance of the QoL comparisons was assessed by controlling the false discovery rate at 5\% (Benjamini and Hochberg, 1995) within each of these sets. Progression-free survival was measured from the date of randomisation to progression or death from any cause (whichever came first). Survival times were also measured from the date of randomisation to death from any cause. Kaplan-Meier methods were used to generate survival curves and estimate survival rates.

\section{RESULTS}

\section{Patients}

Between September 2000 and January 2002, 132 patients were recruited and randomised (44 to each treatment arm) from 11 centres in UK and four centres in Switzerland. The arms were well balanced with respect to baseline demographic and disease characteristics (Table 1).

\section{Treatment and completion rate}

A total of 31 (70.5\%; 90\% confidence interval (CI): $57.2-81.6 \%), 32$ (72.7\%; 90\% CI: 59.6-83.4\%) and 20 (45.5\%; 90\% CI: 32.5-58.9\%) patients completed the full eight cycles (24 weeks) of planned

Table I Patient baseline characteristics

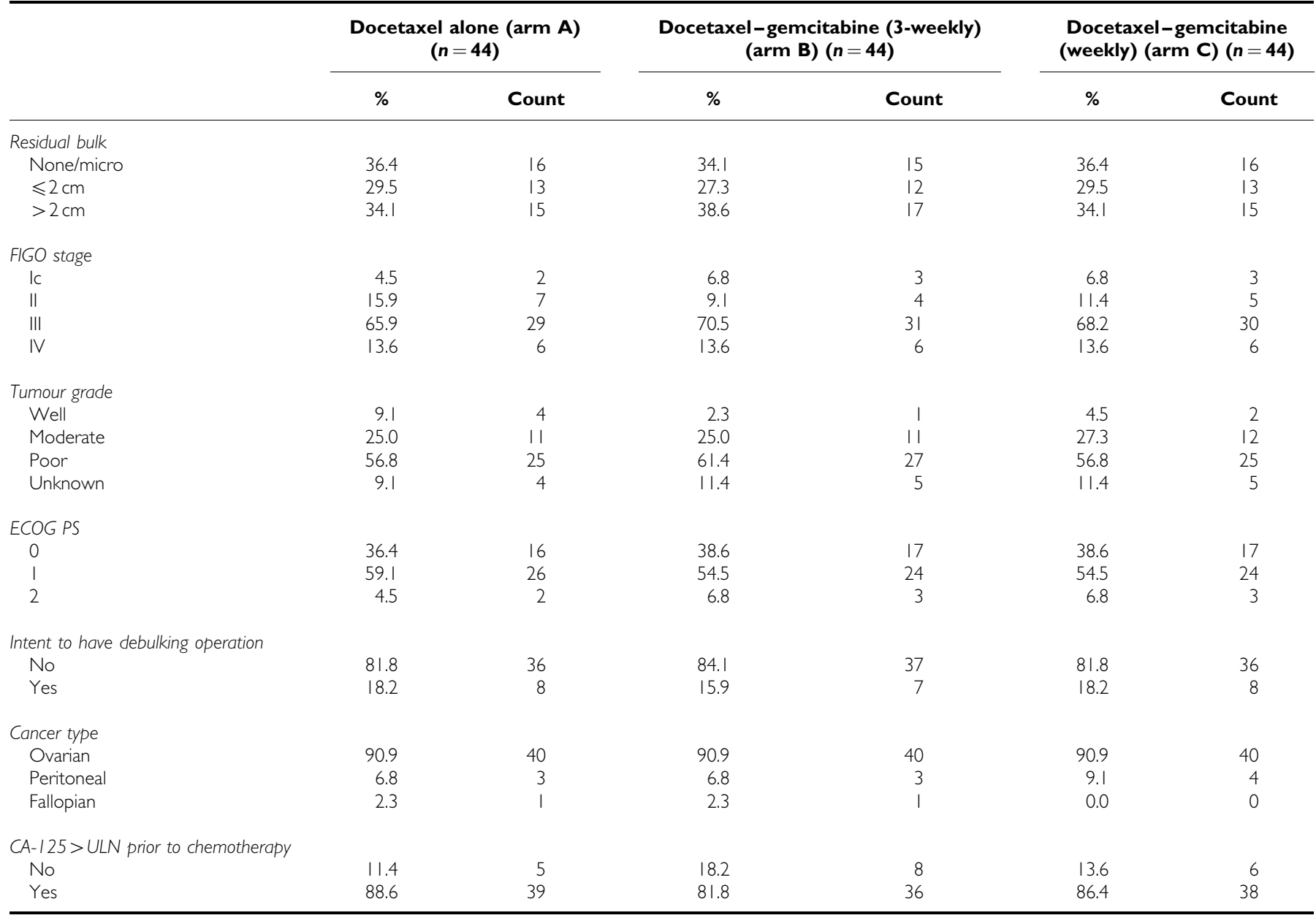

CA-125 = cancer antigen 125; ECOG PS = Eastern Cooperative Oncology Group Performance Status; FIGO = International Federation of Gynecologic Oncology; ULN = upper limit of normal. 


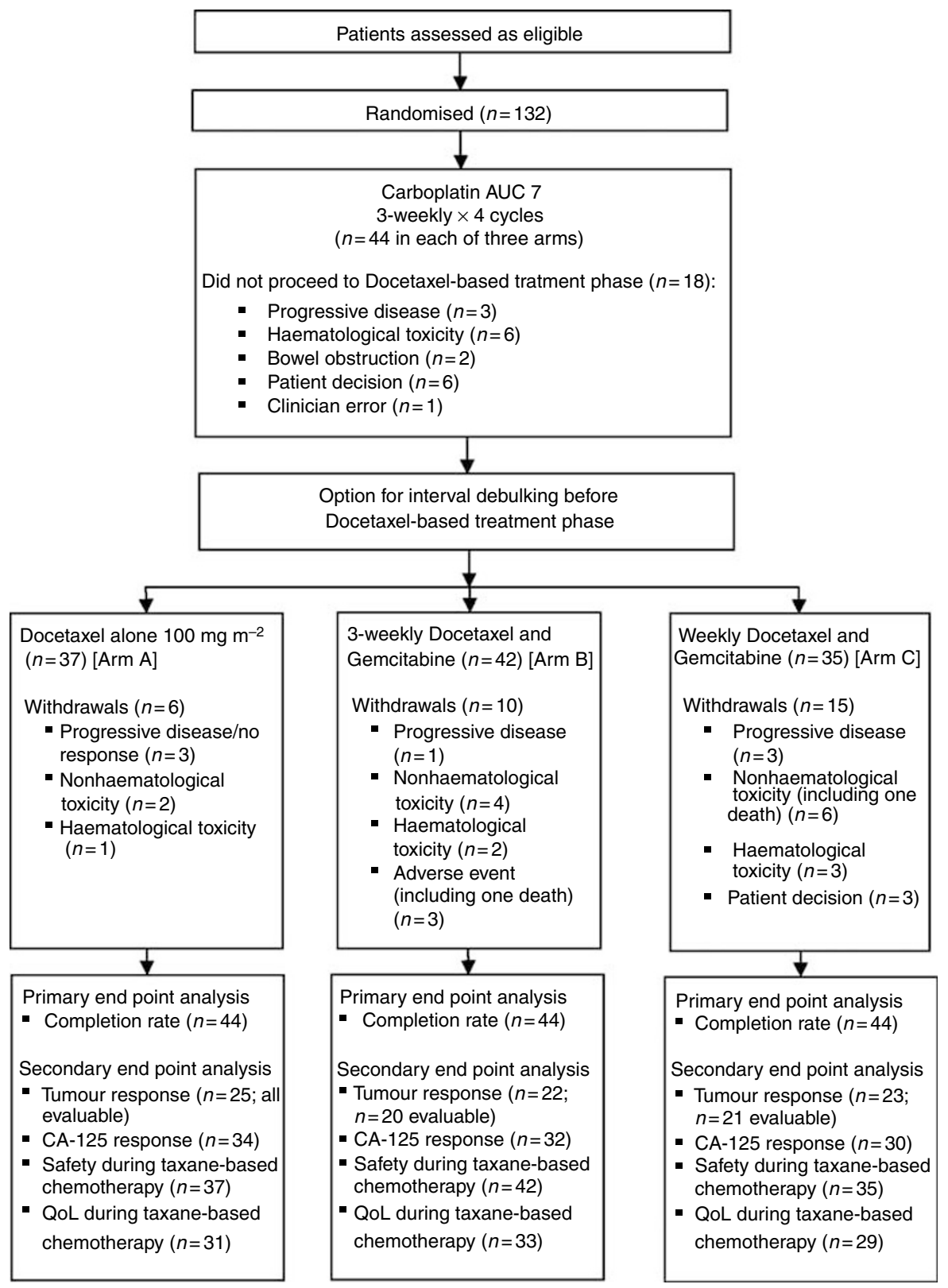

Figure I Patient journey through trial.

treatment in arms A, B and C, respectively (primary end point). None of the arms demonstrated a completion rate that was statistically greater than $60 \%(P=0.102, P=0.056, P=0.982)$, which was our formal feasibility criteria; the completion rate in arm C is markedly less than this level.

The progress of patients through the trial is shown in Figure 1. Overall, $121(91.7 \%)$ patients completed their initial four cycles of carboplatin treatment, where 362 out of 505 (71.7\%) cycles were delivered on time; $48(36.4 \%)$ patients had no delays, while 41 (31.1\%) experienced a delay of one cycle. In most cases (86 (65.2\%) patients), the planned dose of carboplatin was given and only 12 (9.1\%) patients had two or more dose reductions, resulting in a median dose intensity of $92 \%$.

Of the patients who proceeded to docetaxel-based therapy, 31 out of $37(83.8 \%)$ patients, 33 out of $42(78.6 \%)$ patients and 20 out of $35(57.1 \%)$ patients completed four cycles of treatment in arms A, B and C, respectively (Figure 1). In arm A, 17 (45.9\%) patients had no cycle delays, $16(43.2 \%)$ patients had one cycle delay and four $(10.8 \%)$ patients had two cycle delays. In arm B, nine $(21.4 \%)$ patients had no cycle delays and $31(73.8 \%)$ had one to four delays either on day 1 or day 8 of treatment. In arm C, only two $(5.7 \%)$ patients received all cycles on time and 25 (71.4\%) experienced one to four delays.

Dose intensities during docetaxel/gemcitabine chemotherapy were calculated over the period following these agents' first cycle; delays starting docetaxel/gemcitabine as a result of prior carboplatin therapy are not included. The median dose intensities were 99\% (docetaxel in arm A), 84\% (docetaxel in arm B), $86 \%$ (gemcitabine in arm B) and $73 \%$ (for both gemcitabine and doceaxel in arm $\mathrm{C}$ ). The protocol amendment resulted in the dose intensity in arm C increasing from 55 to $74 \%$ (gemcitabine) and 55 to $73 \%$ (docetaxel).

In total, $24(64.9 \%)$ patients in arm A received the full dose at each cycle and $12(32.4 \%)$ patients had one dose reduction. Full dose was given as planned to most patients in arm B, with 30 (71.4\%) and $33(78.6 \%)$ patients receiving full doses of docetaxel and gemcitabine, respectively. In this treatment arm, nine $(21.4 \%)$ patients experienced one docetaxel dose reduction and four $(9.5 \%)$ 
had one gemcitabine dose reduction. Dose reductions were more common in arm C, with only $17(48.6 \%)$ patients having no dose reductions and $14(40.0 \%)$ patients having one dose reduction.

\section{Response}

Clinical response data were available for 70 patients with measurable disease during the carboplatin phase of the study, although seven became unevaluable for various nonclinical reasons (e.g. wrong investigation performed). The overall response rate after this phase was $65.7 \%$ (46 out of 70 ), including $14(20.0 \%)$ complete and $32(45.7 \%)$ partial responses. A total of $12(17.1 \%)$ patients had stable disease and five $(7.1 \%)$ patients had progressive disease. Data on tumour responses at the end of docetaxel-based treatment were available for 25,22 and 23 patients with measurable disease in arms A, B and C, respectively, although four patients subsequently became unevaluable (Table 2 ). The overall response rates after all planned chemotherapy were $84 \%$ (10 complete and 11 partial responses), $77.3 \%$ (10 complete and seven partial responses) and $69.6 \%$ (seven complete and nine partial responses) in arms A, B and C, respectively. Of 96 patients evaluable for CA125 response, $72(75.0 \%)$ responded - most (68 out of 72 ) before docetaxel-based therapy began. Overall, $62.5 \%$ (10 out of 16 patients) in arm A, 100.0\% (11 out of 11 ) in arm B and $87.5 \%$ (14 out of 16) in arm $\mathrm{C}$ had disease which remained stable or improved after the carboplatin phase with the addition of four cycles of docetaxel-based therapy.

\section{Survival}

At the time of this analysis, 86 patients had progressed or died. A total of $79 \%$ of surviving patients had a minimum follow-up of 2 years and the median follow-up was 30 months. The 8 -month progression-free survival rate was $77.3 \%$ (standard error (SE) 6\%) in arm $\mathrm{A}, 93.1 \%$ (SE 4\%) in arm B and 76.9\% (SE 6\%) in arm C; the corresponding median progression-free survival times are 15.5 (95\% CI: 10.5-20.6), 18.1 (95\% CI: 15.9-20.3) and 13.7 (95\% CI: 12.8 - 14.6) months, respectively.

\section{Toxicity}

Table 3 shows the incidence of grade 3-4 haematological toxicity and neutropenic complications occurring during docetaxel-based therapy. Anaemia was more severe in the gemcitabine-containing arms $(P=0.002)$, as was thrombocytopenia $(P<0.001)$. Neutropenia was more severe in the docetaxel-alone arm $(P=0.015)$. No patients had complicated thrombocytopenia. Table 3 also shows the incidence of grade 2-4 nonhaematological toxicity that occurred during docetaxel-based therapy. Alopecia was markedly less in arm C $(P=0.001)$. Dyspnoea was markedly increased in both gemcitabine-containing arms $(P=0.001)$, but was worse with the weekly regimen (arm C). Furthermore, arm $C$ was associated with pulmonary infiltrates in three patients and one death due to pulmonary toxicity. There was one further on-study death due to neutropenic sepsis, which also occurred in arm C.

\section{Quality of life}

Quality of life data were available for 31,33 and 29 patients in arms A, B and C, respectively. Median changes in selected QoL measures during docetaxel-based treatment (average recorded during docetaxel-based therapy minus the value at the end of carboplatin treatment) were compared between the treatment arms. After adjustment for multiple testing, the only statistically significant difference was in nausea and vomiting, which was higher in arm $\mathrm{C}$ (median change 5.6; range -17.0 to 50$)$ compared to arms A (0; -100 to 20$)$ and $\mathrm{B}(0 ;-44$ to 33$)$.

\section{DISCUSSION}

Sequential chemotherapy may potentially maximise the impact of each chemotherapeutic agent while avoiding overlapping toxicities caused by concurrent administration. Following the results of ICON-3 and GOG-132, this represents a logical next step in the evolution of induction chemotherapy for ovarian cancer (Muggia, 2003). Our results suggest that sequential chemotherapy with four cycles of carboplatin AUC 7 followed by a 3-weekly schedule of docetaxel-based chemotherapy is feasible as first-line treatment for patients with ovarian cancer. Carboplatin followed by weekly docetaxel-gemcitabine (arm C) was not well tolerated, and dose intensity was compromised as evidenced by more dose delays, dose reductions and a high rate of discontinuations during the docetaxel-based phase (43\%). Furthermore, the weekly docetaxelgemcitabine arm clearly failed the minimum criterion for feasibility ( $>60 \%$ completion rate for eight cycles). Although the lower end of the $90 \%$ CIs for completion rates in the docetaxelalone and 3-weekly docetaxel-gemcitabine arms was just below $60 \%$, their observed completion rates are sufficiently high to make them worthy of consideration for further clinical testing. Comparisons with toxicity seen in other trials and schedules are fraught with imprecision, but the concurrent triplet combination of carboplatin/paclitaxel/gemcitabine appears to produce significant bone marrow suppression which may make this difficult to deliver consistently in the nontrial setting (reviewed by Hansen, 2002).

Dyspnoea was markedly increased in both gemcitabine-containing arms; however, it was much more clinically significant in the weekly docetaxel-gemcitabine arm, which was also associated with pulmonary infiltration in three patients and one death due to pulmonary toxicity. Lung toxicity has been reported with other weekly taxane-gemcitabine schedules - for example, in the SCOTROC 2C trial, where patients were treated with four cycles of carboplatin followed by weekly paclitaxel-gemcitabine in patients with ovarian cancer (Harries et al, 2004). One trial with paclitaxel plus weekly gemcitabine was discontinued as four out of 12 patients with non-small-cell lung cancer experienced dose-

Table 2 Tumour responses after eight cycles of chemotherapy (by treatment arm)

\begin{tabular}{|c|c|c|c|c|c|c|}
\hline \multirow[b]{2}{*}{ Response } & \multicolumn{2}{|c|}{$\begin{array}{l}\text { Carboplatin followed by docetaxel } \\
\text { alone } \\
\qquad(\operatorname{arm} \text { A) }(n=25)\end{array}$} & \multicolumn{2}{|c|}{$\begin{array}{l}\text { Carboplatin followed by docetaxel-gemcitabine } \\
\qquad \text { (3-weekly) (arm B) }(n=22)\end{array}$} & \multicolumn{2}{|c|}{$\begin{array}{c}\text { Carboplatin followed by } \\
\text { docetaxel-gemcitabine (weekly) } \\
(\operatorname{arm} \text { C) }(n=23)\end{array}$} \\
\hline & $\%$ & Count & $\%$ & Count & $\%$ & Count \\
\hline$C R$ & 40.0 & 10 & 45.5 & 10 & 30.4 & 7 \\
\hline PR & 44.0 & | | & 31.8 & 7 & 39.1 & 9 \\
\hline Stable & 8.0 & 2 & 9.1 & 2 & 8.7 & 2 \\
\hline Progression & 8.0 & 2 & 4.5 & I & 13.0 & 3 \\
\hline Unevaluable & 0.0 & 0 & 9.1 & 2 & 8.7 & 2 \\
\hline
\end{tabular}

$\mathrm{CR}=$ complete response; $\mathrm{PR}=$ partial response. 
Table 3 Haematological (grade 3-4) and nonhaematological (grade 2-4) toxicities (worst grade over cycles of docetaxel-based chemotherapy received)

\begin{tabular}{|c|c|c|c|c|c|c|c|}
\hline \multirow[b]{2}{*}{ Toxicity } & \multirow[b]{2}{*}{ Grade } & \multicolumn{2}{|c|}{$\begin{array}{c}\text { Docetaxel alone } \\
(\operatorname{arm} A)\end{array}$} & \multicolumn{2}{|c|}{$\begin{array}{c}\text { Docetaxel-gemcitabine } \\
\text { (3-weekly) (arm B) }\end{array}$} & \multicolumn{2}{|c|}{$\begin{array}{c}\text { Docetaxel-gemcitabine } \\
\text { (weekly) (arm C) }\end{array}$} \\
\hline & & $\%$ & Count & $\%$ & Count & $\%$ & Count \\
\hline \multicolumn{8}{|l|}{ Haematological } \\
\hline \multirow[t]{2}{*}{ Neutropenia } & 3 & $|1|$. & 4 & 38.1 & 16 & 22.9 & 8 \\
\hline & 4 & 66.7 & 24 & 47.6 & 20 & 28.6 & 10 \\
\hline \multirow{2}{*}{ White blood cells } & 3 & 40.5 & 15 & 40.5 & 17 & 42.9 & 15 \\
\hline & 4 & 24.3 & 9 & 7.1 & 3 & 5.7 & 2 \\
\hline \multirow[t]{2}{*}{ Platelets } & 3 & 8.1 & 3 & 4.8 & 2 & 11.4 & 4 \\
\hline & 4 & 0.0 & 0 & 4.8 & 2 & 0.0 & 0 \\
\hline \multirow[t]{2}{*}{ Haemoglobin } & 3 & 0.0 & 0 & 9.5 & 4 & 8.6 & 3 \\
\hline & 4 & 0.0 & 0 & 2.4 & I & 2.9 & I \\
\hline \multicolumn{8}{|l|}{ Nonhaematological } \\
\hline Alopecia & 2 & 73.0 & 27 & 64.3 & 27 & 19.8 & 7 \\
\hline \multirow[t]{2}{*}{ Nausea } & 2 & 13.5 & 5 & 9.5 & 4 & 28.6 & 10 \\
\hline & 3 & 0.0 & 0 & 7.1 & 3 & 2.9 & I \\
\hline \multirow[t]{2}{*}{ Vomiting } & 2 & 8.1 & 3 & 2.4 & I & 11.4 & 4 \\
\hline & 3 & 0.0 & 0 & 4.8 & 2 & 2.9 & I \\
\hline \multirow[t]{2}{*}{ Sensory neuropathy } & 2 & 13.5 & 5 & 4.8 & 2 & 5.7 & 2 \\
\hline & 3 & 2.7 & 1 & 0.0 & 0 & 0.0 & 0 \\
\hline \multirow[t]{2}{*}{ Motor neuropathy } & 2 & 2.7 & 1 & 0.0 & 0 & 0.0 & 0 \\
\hline & 3 & 0.0 & 0 & 2.4 & 1 & 2.9 & 1 \\
\hline \multirow[t]{2}{*}{ Diarrhoea } & 2 & 16.2 & 6 & 11.9 & 5 & 14.3 & 5 \\
\hline & 3 & 2.7 & 1 & 7.1 & 3 & 0.0 & 0 \\
\hline \multirow[t]{2}{*}{ Constipation } & 2 & 16.2 & 6 & 9.5 & 4 & $1 \mid .4$ & 4 \\
\hline & 3 & 0.0 & 0 & 2.4 & 1 & 5.7 & I \\
\hline \multirow[t]{2}{*}{ Stomatitis } & 2 & 29.7 & $1 \mid$ & 21.4 & 9 & 2.9 & I \\
\hline & 3 & 5.4 & 2 & 0 & 0 & 0.0 & 0 \\
\hline \multirow[t]{3}{*}{ Shortness of breath } & 2 & 10.8 & 4 & 35.7 & 15 & 40.0 & 14 \\
\hline & 3 & 0.0 & 0 & 4.8 & 2 & 5.7 & 2 \\
\hline & 4 & 0.0 & 0 & 0.0 & 0 & 2.9 & 1 \\
\hline \multirow[t]{3}{*}{ Abdominal pain or cramping } & 2 & 10.8 & 4 & 4.8 & 2 & 8.6 & 3 \\
\hline & 3 & 0.0 & 0 & 2.4 & 1 & 5.7 & 2 \\
\hline & 4 & 0.0 & 0 & 2.4 & 1 & 0.0 & 0 \\
\hline \multirow[t]{2}{*}{ Fatigue (lethargy, malaise, asthenia) } & 2 & 43.2 & 16 & 48.6 & 18 & 51.4 & 18 \\
\hline & 3 & 5.4 & 2 & 2.7 & | & 17.1 & 6 \\
\hline
\end{tabular}

limiting pneumonitis (Thomas et al, 2000). While both docetaxel and gemcitabine can be associated with pulmonary toxicities, it was the weekly schedule that appeared to be associated with the development of pulmonary toxicity in the present trial. Although this regimen led to one fatality, this toxicity was otherwise reversible (resolving with time and corticosteroid use). Further evaluation of this toxicity should be undertaken, as it could impact on protocol design for weekly combinations of these agents.

Sequential scheduling with a platinum agent followed by a taxane - with or without other chemotherapeutic agents - has been investigated in several early phase studies in ovarian cancer. Unacceptable neurotoxicity with sequential cisplatin $\left(100 \mathrm{mg} \mathrm{m}^{-2}\right.$ for four cycles) and paclitaxel $\left(200 \mathrm{mg} \mathrm{m}^{-2}\right.$ for four cycles) has been reported (Poole et al, 2000), although in most cases sequential scheduling allowed planned dose intensity to be achieved (Tognoni et al, 2000; Guppy et al, 2004). Importantly, sequential scheduling did not incur loss of efficacy; in these studies, median overall survival ranged from 22 to 30 months (Poole et al, 2000; Tognoni et al, 2000; Guppy et al, 2004). In SCOTROC $2 \mathrm{C}$, the response rate in evaluable patients was $84 \%$, with a median progression-free survival of 19.5 months at a median follow-up of 28 months (Harries et al, 2004). In our study, the response rate of $66 \%$ after single-agent carboplatin was improved following sequential treatment with docetaxel-based regimens. Carboplatin followed by docetaxel alone (arm A) produced the highest response rate $(84 \%$, compared with $77 \%$ in arm B and $70 \%$ in arm C). However, carboplatin followed by 3weekly docetaxel-gemcitabine ( $\operatorname{arm} \mathrm{B})$ was associated with the best-observed survival outcome, with a median progression-free survival time of 18.1 months (95\% CI: 15.9-20.3) - compared with 15.5 months (95\% CI: $10.5-20.6)$ for arm A and 13.7 months $(95 \%$ CI: $12.8-14.6)$ for arm C.

In conclusion, although just failing our formal feasibility criteria, we believe that, all taken together, the observed data for sequential chemotherapy with four cycles of carboplatin followed by 3 -weekly docetaxel-based therapy suggests that this is a realistic option for first-line therapy of ovarian cancer. Preliminary results of the SCOTROC 2B study, which investigated carboplatin followed by docetaxel alone or 3-weekly docetaxel-irinotecan (Jayson et al, 2003), further support this sequential approach and suggest further investigation in ovarian cancer is warranted. Following the results of SCOTROC 2C (Harries et al, 2004), in which pulmonary toxicity was noted with the weekly paclitaxel-gemcitabine schedule, a further feasibility study with the 3-weekly paclitaxel-gemcitabine schedule has also been initiated. We aim to analyse the results of all the SCOTROC 2 trials together (including docetaxel and paclitaxel-based schedules) in order to evaluate the optimal sequence and drug combination for future Phase III testing of a sequential approach to standard, concurrent two-drug chemotherapy administration.

\section{ACKNOWLEDGEMENTS}

This trial was funded by grants from Aventis Pharma and Eli Lilly and Company, who also supplied Taxotere ${ }^{\circledR}$ and Gemzar ${ }^{\mathbb{R}}$, respectively. 


\section{REFERENCES}

American Cancer Society. Cancer Facts \& Figures 2004 (2004). Atlanta, GA: American Cancer Society

Barlow C, Nystrom M, Oesterling C, Fennell D, Ismay J, Gallagher C (2004) Dose intense triplet chemotherapy with gemcitabine, carboplatin, paclitaxel with peripheral blood progenitor cell support for six cycles in advanced epithelial ovarian cancer. $\mathrm{Br} J$ Cancer 90: $1318-1322$

Benjamini Y, Hochberg Y (1995) Controlling the false discovery rate: a practical and powerful approach to multiple testing. J Roy Statist Soc Ser B 57: 289-300

Berkenblit A, Tung N, Kim Y, Feyler H, Niloff J, Berghe KV, Cannistra SA (2003) Phase I trial of docetaxel, carboplatin, and gemcitabine as firstline therapy for patients with high-risk epithelial tumors of müllerian origin. Gynecol Oncol 89: 486-493

Bookman MA (2003) Developmental chemotherapy and management of recurrent ovarian cancer. J Clin Oncol 21(Suppl): 149s-167s

Calvert AH, Newell DR, Gumbrell LA, O’Reilly S, Burnell M, Boxall FE, Siddik ZH, Judson IR, Gore ME, Wiltshaw E (1989) Carboplatin dosage: prospective evaluation of a simple formula based on renal function. J Clin Oncol 7: $1748-1756$

Cassidy J, Paul J, Soukop M, Habeshaw T, Reed NS, Parkin D, Kaye SB (1998) Clinical trials of nimodipine as a potential neuroprotector in ovarian cancer patients treated with cisplatin. Cancer Chemother Pharmacol 41: $161-166$

Cassinelli G, Supino R, Perego P, Polizzi D, Lanzi C, Pratesi G, Zunino F (2001) A role for loss of p53 function in sensitivity of ovarian carcinoma cells to taxanes. Int J Cancer 92: $738-747$

Chantler C, Garnett ES, Parsons V, Veall N (1969) Glomerular filtration rate measurement in man by the single injection methods using ${ }^{51} \mathrm{Cr}$-EDTA. Clin Sci 37: $169-180$

Cull A, Howat S, Greimel E, Waldenstrom AC, Arraras J, Kudelka A, Chauvenet L, Gould A, on behalf of the EORTC Quality of Life Group (European Organization for Research and Treatment of Cancer) and the Scottish Gynaecological Cancer Trials Group (2001) Development of a European Organization for Research and Treatment of Cancer questionnaire module to assess the quality of life of ovarian cancer patients in clinical trials: a progress report. Eur J Cancer 37: $47-53$

Gligorov J, Lotz J-P (2004) Preclinical pharmacology of the taxanes: implications of the differences. Oncologist 9(Suppl 2): 3-8

Guastalla III JP, Dieras V (2003) The taxanes: toxicity and quality of life considerations in advanced ovarian cancer. $\mathrm{Br} J$ Cancer 89(Suppl 3): $\mathrm{S} 16-\mathrm{S} 22$

Guppy AE, Nelstrop AE, Foster T, Agarwal R, Seckl MJ, Rustin GJ (2004) A phase II study of sequential carboplatin, paclitaxel and topotecan in patients with previously untreated advanced ovarian cancer. $\mathrm{Br} J$ Cancer 90: $810-814$

Hansen SW (2002) Gemcitabine, platinum, and paclitaxel regimens in patients with advanced ovarian carcinoma. Semin Oncol 29(1 Suppl 1): $17-19$

Harries M, Moss C, Perren T, Gore M, Hall G, Everard M, A'Hern R, Gibbens I, Jenkins A, Shah R, Cole C, Pizzada O, Kaye S (2004) A phase II feasibility study of carboplatin followed by sequential weekly paclitaxel and gemcitabine as first-line treatment for ovarian cancer. $\mathrm{Br} \mathrm{J}$ Cancer 91: $627-632$
Hsu Y, Sood AK, Sorosky JI (2004) Docetaxel versus paclitaxel for adjuvant treatment of ovarian cancer: case - control analysis of toxicity. Am J Clin Oncol 27: 14-18

International Collaborative Ovarian Neoplasm (ICON) Group (2002) Paclitaxel plus carboplatin versus standard chemotherapy with either single-agent carboplatin or cyclophosphamide, doxorubicin, and cisplatin in women with ovarian cancer: the ICON3 randomised trial. Lancet 360: $505-515$

Jayson GC, Maenpaa J, Wilkinson PM, Ledermann JA, Welch RS Cruickshank D, Chan S, Hindley A, Vasey PA, Fernebro E (2003) Randomized feasibility study of carboplatin followed by docetaxel or docetaxel/irinotecan in ovarian cancer (SCOTROC IIB). Proc Am Soc Clin Oncol 22: 449 (Abstract)

Kaye SB (2000) Intravenous chemotherapy for ovarian cancer - the state of the art? Int J Gynecol Cancer 10: 19-25

Lavarino C, Pilotti S, Oggionni M, Gatti L, Perego P, Bresciani G, Pierotti MA, Scambia G, Ferrandina G, Fagotti A, Mangioni C, Lucchini V, Vecchione F, Bolis G, Scarfone G, Zunino F (2000) p53 gene status and response to platinum/paclitaxel-based chemotherapy in advanced ovarian carcinoma. J Clin Oncol 18: $3936-3945$

Mäenpää JU (2003) Docetaxel: promising and novel combinations in ovarian cancer. Br J Cancer 89(Suppl 3): S29-S34

Muggia FM (2003) Sequential single agents as first line chemotherapy for ovarian cancer: a strategy derived from the results of GOG132. Int J Gyn Cancer 13(Suppl 2): $156-162$

Muggia FM, Braly PS, Brady MF, Sutton G, Niemann TH, Lentz SL, Alvarez RD, Kucera PR, Small JM (2000) Phase III randomized study of cisplatin versus paclitaxel versus cisplatin and paclitaxel in patients with suboptimal stage III or IV ovarian cancer: a Gynecologic Oncology Group study. J Clin Oncol 18: $106-115$

Poole CJ, Perren T, Burton A, Jordan SD, Jenkins AH, Mould JJ, Spooner DA, Luesley D, Chan KK, Sturman S, Earl HM (2000) Phase II clinical trials of cisplatin-then-paclitaxel and paclitaxel-then-cisplatin in patients with previously untreated advanced epithelial ovarian cancer. Ann Oncol 11: $1603-1608$

Reles A, Wen WH, Schmider A, Gee C, Runnebaum IB, Kilian U, Jones LA, El-Naggar A, Minguillon C, Schonborn I, Reich O, Kreienberg R, Lichtenegger W, Press MF (2001) Correlation of p53 mutations with resistance to platinum-based chemotherapy and shortened survival in ovarian cancer. Clin Cancer Res 7: 2984-2997

Rustin GJS, Nelstrop AE, Tuxen MK, Lambert HE (1996) Defining progression of ovarian carcinoma during follow-up according to CA 125: a North Thames Ovary Group Study. Ann Oncol 7: $361-364$

Thomas AL, Cox G, Sharma RA, Steward WP, Shields F, Jeyapalan K, Muller S, O'Byrne KJ (2000) Gemcitabine and paclitaxel associated pneumonitis in non-small cell lung cancer: report of a phase I/II doseescalating study. Eur J Cancer 36: 2329-2334

Tognoni A, Pronzato P, Cadenotti L, Ghio E, Manna N, Pensa F, Marino L (2000) Sequential high dose-density chemotherapy in advanced ovarian cancer. Anticancer Res 20: 3957 - 3961

Vasey PA, Jayson GC, Gordon A, Gabra H, Coleman R, Atkinson R, Parkin D, Paul J, Hay A, Kaye SB, the Scottish Gynaecological Cancer Trials Group (2004) Phase III randomized trial of docetaxel-carboplatin versus paclitaxel - carboplatin as first-line chemotherapy for ovarian carcinoma. J Natl Cancer Inst 96: 1682-1691 